\title{
A SEMI-BLIND ALGEBRAIC CONSTANT MODULUS ALGORITHM
}

\author{
A. Lee Swindlehurst
}

\author{
Dept. of Electrical \& Computer Engineering \\ Brigham Young University \\ Provo, UT 84604 \\ E-mail: swindle@ee.byu.edu .
}

\begin{abstract}
A modification to the Algebraic Constant Modulus (ACM) algorithm is proposed that exploits the presence of known pilot symbols within the transmitted data. The pilot symbols are used to place soft constraints on the subspace in which the ACM solution should lie, and relax some of the identifiability conditions required by ACM. The primary advantage of the proposed method is that sources transmitting linearly independent pilot data can be separated without resorting to a joint diagonalization procedure, and hence without computing the beamformers of signals that are not of interest.
\end{abstract}

\section{INTRODUCTION}

The Algebraic (or sometimes "Analytic") Constant Modulus (ACM) algorithm [1] provides a closed-form solution to the problem of blindly separating multiple constant modulus (CM) signals. The algorithm finds a set of linear combiners or "beamformers" for all of the CM signals (both desired and interference) that are present in the received data. It is implemented in two steps: First, a least-squares problem is solved in which the quadratic CM constraints are relaxed in order to obtain the estimate of a particular subspace, and second, the CM constraints are applied via a joint-diagonalization procedure performed on the vectors that span the subspace estimate. The excellent performance of ACM has been documented using both analytical performance evaluations $[2,3,4]$ and studies involving experimental data [5].

This paper presents an extension to the ACM approach that allows the receiver to exploit knowledge of pilot or training symbols embedded in at least one of the transmitted signals. This information is used to define a subspace orthogonal to that in which the desired beamformer must lie, which in turn is used to augment the number of equations used in the ACM least-squares problem. Assuming no other source employs the same pilot signal at the same time, a single solution results that separates the desired signal from the other CM signals without the need for finding beamformers for all of the other sources via joint diagonalization. In situations where only one of the received signals is of interest, ACM estimates the waveforms of all of the $\mathrm{CM}$ signals that are present, and must employ additional information (e.g., pilot data) to determine which of the signals corresponds to the desired source. The approach we present takes advantage of information about the pilot data from the beginning, and uses it simultaneously with the CM assumption to find the beamformer of the desired signal directly. The new algorithm is similar in spirit to previous extensions of ACM that exploit structure in the data due to channel memory [6] or space-time block coding [7].

\section{ALGEBRAIC CONSTANT MODULUS ALGORITHM}

Consider an array of $M$ antennas that collects $N$ samples of $D$ CM signals:

$$
\mathbf{X}=\mathbf{H S}
$$

where $\mathbf{X}$ is $M \times N$ and contains the array outputs, $\mathbf{H}$ is $M \times D$ and represents the unknown channel, and $\mathbf{S}$ is $D \times$ $N$ and holds the CM signal samples (the presence of noise is neglected for the moment). The rows of $\mathbf{S}$ are denoted by $\mathbf{s}_{k}^{T}, k=1, \cdots, D$, and the elements in these rows are assumed without loss of generality to have unit modulus: $\left|\mathbf{s}_{k}(i)\right|^{2}=1$ for $i=1, \cdots, N$. Denote the "economy" singular value decomposition (SVD) of $\mathbf{X}$ as follows:

$$
\mathbf{X}=\mathbf{U} \boldsymbol{\Sigma} \mathbf{V}^{*},
$$

where $(\cdot)^{*}$ denotes the complex conjugate transpose and $\mathbf{V}$ is $N \times D$. Note that in the noiseless case,

$$
\text { row } \operatorname{span}(\mathbf{X})=\text { row } \operatorname{span}\left(\mathbf{V}^{*}\right)=\text { row } \operatorname{span}(\mathbf{S}) \text {. }
$$


The goal of the ACM algorithm is to exploit the CM property to find a set of zero-forcing beamformers, $\mathbf{w}_{k}$, that recover each of the signals:

$$
\mathbf{w}_{k}^{*} \mathbf{V}^{*}=\mathbf{s}_{k}^{T},
$$

where $(\cdot)^{*}$ denotes the complex conjugate transpose. As such, the algorithm requires $M \geq D$. Combining (1) together with the CM constraint yields the following set of equations (dropping the subscript $k$ for simplicity):

$\mathbf{w}^{*} \mathbf{v}_{n} \mathbf{v}_{n}^{*} \mathbf{w}=\left(\overline{\mathbf{v}}_{n} \otimes \mathbf{v}_{n}\right)^{*}(\overline{\mathbf{w}} \otimes \mathbf{w})=1, n=1, \cdots, N$,

where $\mathbf{v}_{n}$ is the $n^{\text {th }}$ column of $\mathbf{V}^{*},(\bar{\cdot})$ denotes the complex conjugate and $\otimes$ the Kronecker product. Stacking all such $N$ equations together yields

$$
\left[\begin{array}{c}
\left(\overline{\mathbf{v}}_{1} \otimes \mathbf{v}_{1}\right)^{*} \\
\vdots \\
\left(\overline{\mathbf{v}}_{N} \otimes \mathbf{v}_{N}\right)^{*}
\end{array}\right](\overline{\mathbf{w}} \otimes \mathbf{w}) \stackrel{\text { def }}{=} \mathbf{A}(\overline{\mathbf{w}} \otimes \mathbf{w})=\mathbf{1}_{N},
$$

where $\mathbf{1}_{N}$ is an $N \times 1$ vector of ones. Provided that $N>$ $D^{2}$, equation (3) will have $D$ solutions, each of which corresponds to the beamformer for one of the signals.

In the presence of noise, the ACM algorithm uses the properties described above in the following three-step procedure:

1. Calculate the SVD of $\mathbf{X}$, and define $\mathbf{V}$ to be the $D$ right singular vectors with largest singular values.

2. Form the matrix $\left[\mathbf{A}-\mathbf{1}_{N}\right]$, and estimate its nullspace by the $D$ right singular vectors with smallest singular values.

3. Form a $D \times D$ matrix from each column of the estimated nullspace by inverse column stacking (ignore the last element of each vector), and perform a joint diagonalization of the matrices to calculate the beamformers.

As mentioned earlier, ACM simultaneously provides beamformers for all $D$ signals, whether they correspond to desired signals or not. At this point, some method must be used to determine which of the solutions correspond to the signal(s) of interest. For example, the estimated signals $\mathbf{s}_{k}, k=1, \cdots, D$, could be compared against some known samples of the desired source, and the one "closest" to those samples would then be chosen as the estimate of the desired signal. In the next section, a method is presented that takes advantage of such information from the beginning.

\section{SEMI-BLIND ACM}

Suppose that $T$ samples of one of the CM signals, say $\mathbf{s}_{1}$, are known at the receiver. Denote the vector of known samples as

$$
\mathbf{t}_{1}=\left[\mathbf{s}_{1}\left(i_{1}\right) \cdots \mathbf{s}_{1}\left(i_{T}\right)\right],
$$

where $i_{1}, \cdots, i_{T}$ are the indices of $\mathbf{s}_{1}$ at which these samples occur. Collect the $T$ columns of $\mathbf{V}^{*}$ that correspond to these sample times into a new matrix $\mathbf{V}_{1}^{*}$ :

$$
\mathbf{V}_{1}^{*}=\left[\begin{array}{lll}
\mathbf{v}_{i_{1}} & \cdots & \mathbf{v}_{i_{T}}
\end{array}\right],
$$

so that, in the noiseless case, $\mathbf{V}_{1} \mathbf{w}_{1}=\mathbf{t}_{1}^{*}$, and hence

$$
\mathbf{P}_{t_{1}^{*}}^{\perp} \mathbf{V}_{1} \mathbf{w}_{1} \stackrel{\text { def }}{=} \mathbf{Q}_{1} \mathbf{w}_{1}=0
$$

where $\mathbf{P}_{t_{1}^{*}}^{\perp}=\mathbf{I}-\mathbf{t}_{1}^{*}\left(\mathbf{t}_{1} \mathbf{t}_{1}^{*}\right)^{-1} \mathbf{t}_{1}$, and it is assumed that $T>1$.

Equation (6) provides $T$ additional equations that can be used to augment those already available in (3) from the $\mathrm{CM}$ assumption. To see this, write the $T$ columns of $\mathbf{Q}_{1}^{*}$ as

$$
\mathbf{Q}_{1}^{*}=\left[\begin{array}{lll}
\mathbf{q}_{1} & \cdots & \mathbf{q}_{T}
\end{array}\right]
$$

so that

$$
\left[\begin{array}{c}
\left(\overline{\mathbf{q}}_{1} \otimes \mathbf{q}_{1}\right)^{*} \\
\vdots \\
\left(\overline{\mathbf{q}}_{T} \otimes \mathbf{q}_{T}\right)^{*}
\end{array}\right]\left(\overline{\mathbf{w}}_{1} \otimes \mathbf{w}_{1}\right) \stackrel{\text { def }}{=} \mathbf{A}_{1}\left(\overline{\mathbf{w}}_{1} \otimes \mathbf{w}_{1}\right)=\mathbf{0}_{T},
$$

where $\mathbf{0}_{T}$ is a $T$-element vector of zeros. Combining (7) with (3) yields the following equation for $\mathbf{w}_{1}$ :

$$
\begin{array}{r}
{\left[\begin{array}{cc}
\mathbf{A} & -\mathbf{1}_{N} \\
\alpha \mathbf{A}_{1} & \mathbf{0}_{T}
\end{array}\right]\left[\begin{array}{c}
\overline{\mathbf{w}}_{1} \otimes \mathbf{w}_{1} \\
1
\end{array}\right] \stackrel{\stackrel{\text { def }}{=} \tilde{\mathbf{A}}\left[\begin{array}{c}
\overline{\mathbf{w}}_{1} \otimes \mathbf{w}_{1} \\
1
\end{array}\right](8)}{ }} \\
=\mathbf{0}_{N+T} .
\end{array}
$$

The scaling $\alpha \in \mathbb{R}$ can be used to weight the importance of the pilot data relative to the $\mathrm{CM}$ assumption. As long as no other signal employs the same pilot data, $\mathbf{w}_{1}$ can be almost uniquely determined from (8). "Almost uniquely" refers to the fact that (8) only defines $\mathbf{w}_{1}$ to within some arbitrary phase factor $e^{j \phi}$. If $\mathbf{w}$ denotes any solution to (8), $\phi$ can simply be found by solving

$$
e^{j \phi} \mathbf{V}_{1} \mathbf{w}=\mathbf{t}_{1}^{*} .
$$

The uniqueness of the above solution is reflected by the fact that $\tilde{\mathbf{A}}$ will have only one zero singular value. The first $D^{2}$ elements of the corresponding singular vector will be proportional to $\overline{\mathbf{w}}_{1} \otimes \mathbf{w}_{1}$, and if a $D \times D$ matrix is formed from these elements by inverse column stacking, it will be proportional to the rank-one matrix $\mathbf{W}_{1}=\mathbf{w}_{1} \mathbf{w}_{1}^{*}$. This observation is used in the algorithm description given below.

The relationships derived above suggest the following Semi-Blind ACM (SB-ACM) algorithm when the data is observed in the presence of noise:

1. Calculate the SVD of $\mathbf{X}$, define $\mathbf{V}$ to be the $D$ right singular vectors with largest singular values, and form $\mathbf{V}_{1}$ as in (5). 
2. Construct $\tilde{\mathbf{A}}$ as in (3)-(8) using $\alpha, \mathbf{V}, \mathbf{V}_{1}$, and $\mathbf{P}_{t_{1}^{*}}^{\perp}$, and find the right singular vector of $\tilde{\mathbf{A}}$ corresponding to the smallest singular value.

3. Form a $D \times D$ matrix $\mathbf{W}_{1}$ from the first $D^{2}$ elements of this vector by inverse column stacking, and find the left (or right) singular vector of $\mathbf{W}_{1}+\mathbf{W}_{1}^{*}$ corresponding to the largest singular value. Call this singular vector $\hat{\mathbf{w}}$.

4. Estimate the phase factor $\phi$ using:

$$
\hat{\phi}=\angle\left(\frac{\hat{\mathbf{w}}^{*} \mathbf{t}_{1}}{\hat{\mathbf{w}}^{*} \mathbf{V}_{1}^{*} \mathbf{V}_{1} \hat{\mathbf{w}}}\right)
$$

5. Set $\hat{\mathbf{w}}_{1}=e^{j \hat{\phi}} \hat{\mathbf{w}}$.

In Step 3, the matrix $\mathbf{W}_{1}+\mathbf{W}_{1}^{*}$ is calculated since, when noise is present, $\mathbf{W}_{1}$ will in general not be Hermitian.

The presence of the pilot data provides $T$ equations beyond the $N$ available due to the CM assumption, so the upper bound on the number of separable sources is relaxed to $D^{2}<N+T$. Also, unlike other semi-blind methods or algorithms that use pilot data only, the above approach is able to exploit the availability of known symbols even when $T<D$. Although the computation involved in Step 2 is slightly greater than the corresponding step in the standard ACM algorithm (since $\tilde{\mathbf{A}}$ is larger than $\mathbf{A}$ ), the overall simplicity of the algorithm is improved since no joint diagonalization step is required, and only the beamformer for the signal of interest is computed. A simple simulation example is given in the next section to illustrate the performance of the above algorithm.

\section{A SIMULATION EXAMPLE}

In this example, a single desired QPSK source was simulated in the presence of four non-CM interferers and spatially white noise. The receiver was assumed to have $M=8$ antennas, and the $8 \times 5$ channel matrix $\mathbf{H}$ was composed of independent identically distributed elements. The interference, noise and channel samples were all generated as zeromean complex circular Gaussian random variables. Each block of data was of length $40+T$, the first $T$ symbols of the desired signal were assumed to be known at the receiver, while the remaining 40 were unknown. The desired signal and the interferers each had the same power level, which was $15 \mathrm{~dB}$ higher than that of the additive noise. A total of 100, 000 independent Monte Carlo trials were conducted, each trial with a new realization of the channel, signals and noise. Equation (11) was employed to estimate the proper phase rotation required in order to make symbol decisions using the blind ACM solution. In addition to ACM and

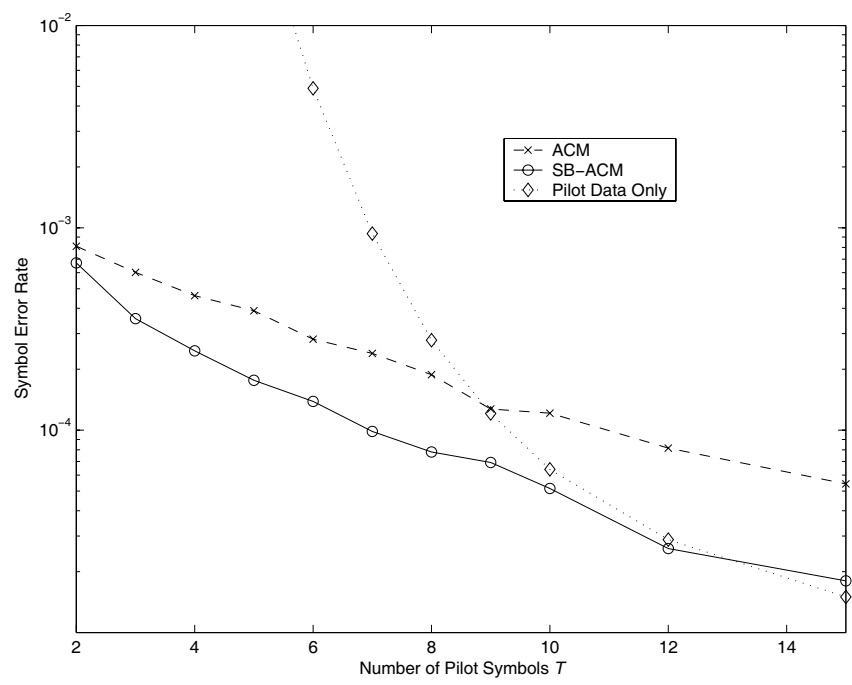

Fig. 1. Symbol Error Rate vs. Number of Pilot Symbols for $N=40, M=8,15 \mathrm{~dB}$ SNR, 4 interfering 15dB Gaussian sources, Rayleigh fading channel.

SB-ACM, results were tabulated for a simple least-squares beamformer based only on the pilot data:

$$
\hat{\mathbf{w}}_{L S}=\left(\mathbf{V}_{1}^{*} \mathbf{V}_{1}\right)^{-1} \mathbf{V}_{1}^{*} \mathbf{t}_{1}^{*}
$$

This approach will only produce a meaningful estimate when $T \geq 5$. In this example, SB-ACM was implemented with no weighting $(\alpha=1)$.

Figure 1 shows the resulting symbol error rate of the three algorithms as $T$ is varied from 2 to 15 . In this example, using $T \geq 9$ pilot symbols as in (12) provides better performance than exploiting the $\mathrm{CM}$ assumption over the entire $40+T$ sample block. However, the SB-ACM approach provides the best performance since it uses knowledge of both the pilot data and the CM property.

\section{CONCLUSIONS}

An generalization of the Algebraic Constant Modulus algorithm has been presented that incorporates information from known pilot symbols embedded in the data. Provided that a given source transmits a unique pilot sequence, it can be separated from interfering sources (CM or otherwise) using a procedure similar to that of the standard ACM algorithm, but without the need for a joint diagonalization step. Only the beamformer for the desired signal, and not the interferers, is computed. The addition of the pilot data also reduces the lower bound on the size of the data block required to achieve source separation. The relative performance of the new algorithm is best in scenarios where only a few pilot symbols are present, or when the size of the overall data 
block is small; situations where exploiting only the pilot data or the $\mathrm{CM}$ assumption alone yield poor or marginal performance.

\section{ACKNOWLEDGEMENTS}

This work was supported by the National Science Foundation under Information Technology Research Grants CCR0081476 and CCR-0313056.

\section{REFERENCES}

[1] A. van der Veen and A. Paulraj, "An Analytical Constant Modulus Algorithm”, IEEE Trans. Sig. Proc., vol. 44, no. 5, pp. 1136-1157, May 1996.

[2] A. van der Veen, "Asymptotic Properties of the Algebraic Constant Modulus Algorithm", IEEE Trans. Sig. Proc., vol. 49, no. 8, pp. 1796-1807, August 2001.

[3] A. van der Veen, "Statistical Performance Analysis of the Algebraic Constant Modulus Algorithm", IEEE Trans. Sig. Proc., vol. 50, no. 12, pp. 3083-3097, December 2002.

[4] B. Sadler, R. Kozick, and T. Moore, "On the Performance of Source Separation with Constant Modulus Signals", in Proc. ICASSP, Orlando, FL, 2002.

[5] A. Swindlehurst, M. Goris, and B. Ottersten, "Some Experiments with Array Data Collected in Actual Urban and Suburban Environments", in Proc. SPAWC Workshop, Paris, France, 1997, pp. 301-304.

[6] A. van der Veen and A. Trindade, "Combining Blind Equalization with Constant Modulus Properties", in Proc. 34th Asilomar Conf. on Signals, Systems, and Computers, 2000, vol. 2, pp. 1568-1572.

[7] A. Swindlehurst, "Blind Separation of Space-Time Block Coded Signals via the Analytic Constant Modulus Algorithm", in Proc. IEEE Sensor Array \& Multichannel Proc. Workshop, Washington, DC, 2002. 